\title{
A Computationally Efficient Algorithm for Computing Convex Hull Prices
}

Bernard Knueven

FERC Technical Conference

24 June 2020

Co-authors: James Ostrowski, Anya Castillo, Jean-Paul Watson 
1 Introduction

2 Primal Formulation \& Decomposition for CHPP

3 Computational Results

4 Analysis of Approximate Convex Hull Prices

5 Conclusion 


\section{Introduction}

\section{Non-convex markets}

- Operating markets with non-convexities poses challenges both theoretical and practical

- Theoretical: Typically no (good) convergence guarantees for associated optimization problems; uniform market-clearing prices may not exist

- Practical: Consequences of clearing the market with a suboptimal solution; which of many pricing mechanisms to use; how to enforce compliance with schedule for participants not correctly incentivized

\section{Convex Hull Pricing (CHP)}

- Minimizes specific side payments

- lost opportunity costs are typically lower with CHP

- Not tied to a (suboptimal) primal solution

- Can lead to counter-intuitive results; e.g., off-line units can be marginal, non-binding transmission constraints in primal solution can have non-zero shadow prices

- Can be difficult to compute in practice 


\section{Introduction}

\section{Non-convexities in Electricity Markets}

- Two main sources: generator operating characteristics (minimum stable power level, minimum run/stop time, start-up costs) and AC power flow

- Market operators in the US typically linearize power flow constraints but not generator operating characteristics

- Medium- to long-term scheduling in US markets therefore involves solving a mixed-integer linear programming problem

- This work considers the convex hull pricing problem (CHPP) in this context 


\section{Contribution}

\section{Solving the Convex Hull Pricing Problem (CHPP)}

- Hua and Baldick (2017) proved a primal linear programming problem could solve CHPP if explicit representations of the convex hull for all generating units is known

- Such linear programs can be very large - the best-known convex hull formulation for a generator grows cubically with the number of time periods

- If some extra constraints, like daily maximum power are present, the best know convex hull formulation is exponential in the number of time steps

- Alvarez et al. (2019) and Yu et al. (2020) propose algorithms which are heuristics when generators have binding ramping limits

- This work proposed a Benders decomposition approach for solving CHPP, leveraging both recent generator convex hull developments and recent advancements in tight and compact formulations for UC (K. et al. 2020) 
Primal Formulation \&
Decomposition for CHPP

Primal Formulation \&
Decomposition for CHPP 


\section{Primal Formulation for CHPP}

\section{Unit Commitment}

$$
\min \sum_{g \in \mathcal{G}} c^{g}
$$

$\sum_{g \in \mathcal{G}}\left(A^{g} p^{g}+B^{g} u^{g}\right)+N(s)=D$

$\left(u^{g}, p^{g}, c^{g}\right) \in \Pi^{g}, \forall g \in \mathcal{G}$

\section{Primal CHPP}

$$
\min \sum_{g \in \mathcal{G}} c^{g}
$$

$$
\begin{aligned}
& \sum_{g \in \mathcal{G}}\left(A^{g} p^{g}+B^{g} u^{g}\right)+N(s)=D \\
& \left(u^{g}, p^{g}, c^{g}\right) \in \operatorname{conv}\left(\Pi^{g}\right), \quad \forall g \in \mathcal{G} .
\end{aligned}
$$

Explicit formulations of the convex hull for every market participant, as required for the Primal CHPP, typically make the approach intractable for large systems. Recent research (Frangioni \& Gentile 2015, K. et al. 2018, Guan et al. 2018) makes this less of an issue for the prototypical thermal generator. 


\section{Primal Formulation for CHPP}

\section{Unit Commitment}

$$
\min \sum_{g \in \mathcal{G}} c^{g}
$$

\section{Primal aCHPP}

$$
\min \sum_{g \in \mathcal{G}} c^{g}
$$

$$
\begin{array}{ll}
\sum_{g \in \mathcal{G}}\left(A^{g} p^{g}+B^{g} u^{g}\right)+N(s)=D & \sum_{g \in \mathcal{G}}\left(A^{g} p^{g}+B^{g} u^{g}\right)+N(s)=D \quad\left(\pi^{\mathrm{aCHP}(\mathcal{R})}\right) \\
\left(u^{g}, p^{g}, c^{g}\right) \in \Pi^{g}, \forall g \in \mathcal{G} & \left(u^{g}, p^{g}, c^{g}\right) \in \mathcal{R}\left(\Pi^{g}\right), \forall g \in \mathcal{G} .
\end{array}
$$

One way around this issue is to instead compute "approximated" convex hull prices. Here $\mathcal{R}\left(\Pi^{g}\right)$ is some MILP relaxation for the set $\Pi^{g}$. 


\section{Primal Formulation for CHPP}

Primal CHPP

$\min \sum_{g \in \mathcal{G}} c^{g}$

\section{Primal aCHPP}

$$
\min \sum_{g \in \mathcal{G}} c^{g}
$$

$$
\begin{array}{ll}
\sum_{g \in \mathcal{G}}\left(A^{g} p^{g}+B^{g} u^{g}\right)+N(s)=D \quad\left(\pi^{\mathrm{CH}}\right) & \sum_{g \in \mathcal{G}}\left(A^{g} p^{g}+B^{g} u^{g}\right)+N(s)=D \quad\left(\pi^{\mathrm{aCHP}(\mathcal{R})}\right) \\
\left(u^{g}, p^{g}, c^{g}\right) \in \operatorname{conv}\left(\Pi^{g}\right), \forall g \in \mathcal{G} . & \left(u^{g}, p^{g}, c^{g}\right) \in \mathcal{R}\left(\Pi^{g}\right), \forall g \in \mathcal{G}
\end{array}
$$

Because $\mathcal{R}\left(\Pi^{g}\right) \subseteq \operatorname{conv}\left(\Pi^{g}\right)$, the Primal aCHPP problem is a relaxation of the Primal CHPP problem. It follows that $\mathcal{R}\left(\Pi^{g}\right)$ can be added to the Primal CHPP problem and not change the feasible region. 


\section{Primal Projected/Benders Formulation for CHPP}

\section{Primal CHPP (redundant constraints/EF)}

$\sum_{g \in \mathcal{G}}\left(A^{g} p^{g}+B^{g} u^{g}\right)+N(s)=D \quad\left(\pi^{\mathrm{CH}}\right)$

$\left(u^{g}, p^{g}, c^{g}\right) \in \operatorname{conv}\left(\Pi^{g}\right), \forall g \in \mathcal{G}$

$\left(u^{g}, p^{g}, c^{g}\right) \in \mathcal{R}\left(\Pi^{g}\right), \forall g \in \mathcal{G}$.

$$
\min \sum_{g \in \mathcal{G}} c^{g}
$$

Primal Projected/Benders CHPP

$$
\min \sum_{g \in \mathcal{G}} c^{g}
$$

$$
\begin{aligned}
& \sum_{g \in \mathcal{G}}\left(A^{g} p^{g}+B^{g} u^{g}\right)+N(s)=D \quad\left(\pi^{\mathrm{CH}}\right) \\
& \alpha_{g, i}^{T} u^{g}+\beta_{g, i}^{T} p^{g}+\delta_{g, i}^{T} c^{g} \leq \eta_{g, i}, \forall i \in \mathcal{F}_{g}, \forall g \in \mathcal{G} \\
& \left(u^{g}, p^{g}, c^{g}\right) \in \mathcal{R}\left(\Pi^{g}\right), \quad \forall g \in \mathcal{G} .
\end{aligned}
$$

Here the set $\mathcal{F}_{g}$ can be considered as the projection of $\operatorname{conv}\left(\Pi^{g}\right)$ onto the $\left(u^{g}, p^{g}, c^{g}\right)$ variables, or in the Benders context, the subset of the projection (along with $\mathcal{R}\left(\Pi^{g}\right)$ ) sufficient to ensure $\left(u^{g}, p^{g}, c^{g}\right) \in \operatorname{conv}\left(\Pi^{g}\right)$. 


\section{Benders Decomposition for CHPP}

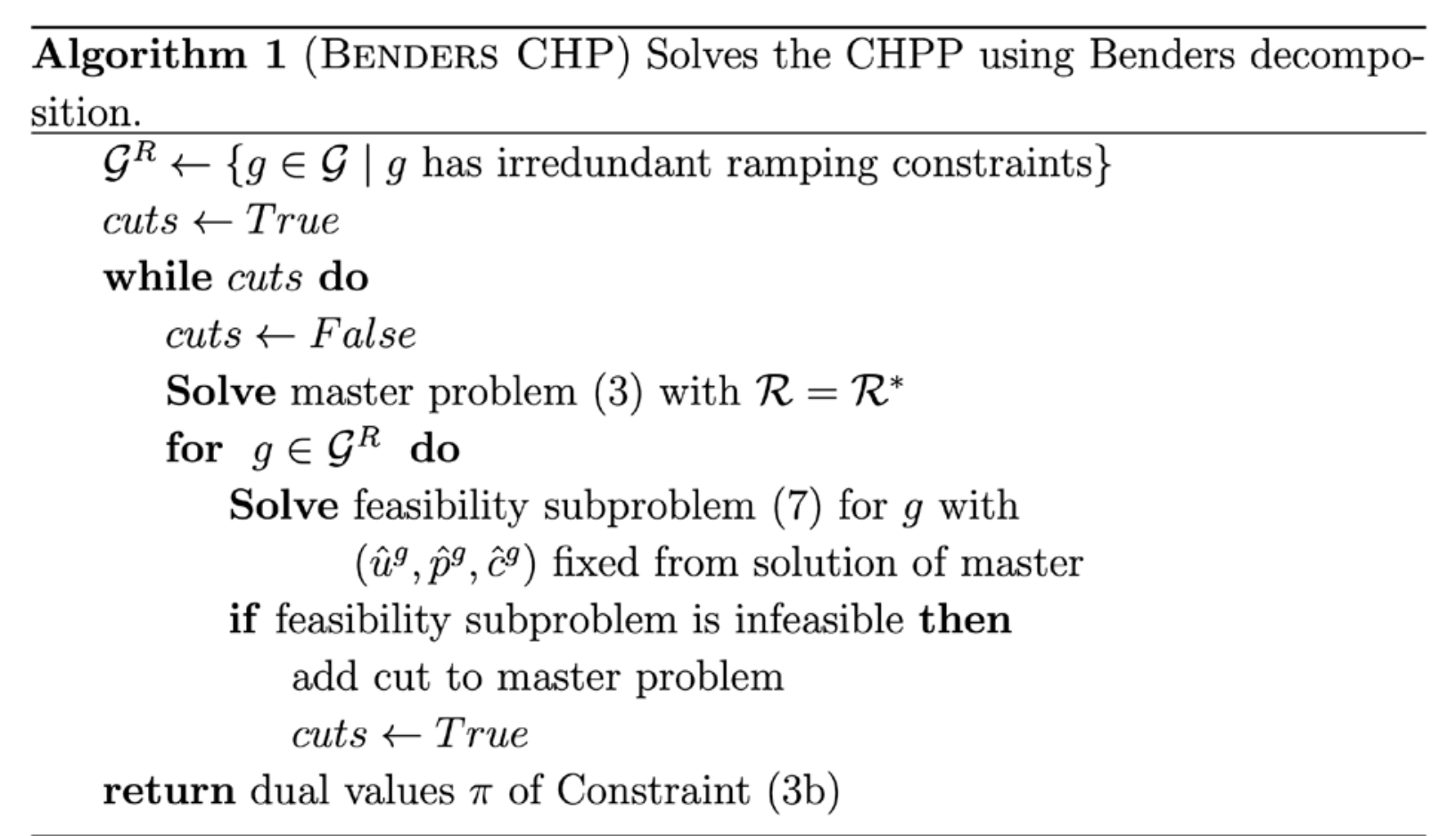

Theorem 2 (Benders Procedure[13]). If $\operatorname{conv}\left(\Pi^{g}\right)$ is a polytope for every $g \in \mathcal{G}$, and the CGLP solver returns vertex solutions, then Algorithm 1 converges in a finite number of iterations.

Theorem 3. The price, $\pi$, returned by Algorithm 1 is a convex hull price. That is, there exists an optimal solution $\left(\pi^{\text {conv }}, \lambda^{\text {conv }}\right)$ to the dual of the EF where $\pi^{\text {conv }}=\pi$. 
Computational Results 


\section{Test Instances \& Platform}

Test instances: pglib-uc (https://github.com/power-grid-lib/pglib-uc)

- 24 \& 48 hourly time steps

- Three sets of instances, ferc, ca, rts_gmlc

- Number of generators

- ferc: 900, ca: $\sim 600$, rts_gmlc: 100

\section{Platform}

- Gurobi used to solve all LPS

- 24-hour instances: MacBook Pro

- 48-hour instances: 64-core Linux workstation 


\section{Computational Results: 24-hour Instances}

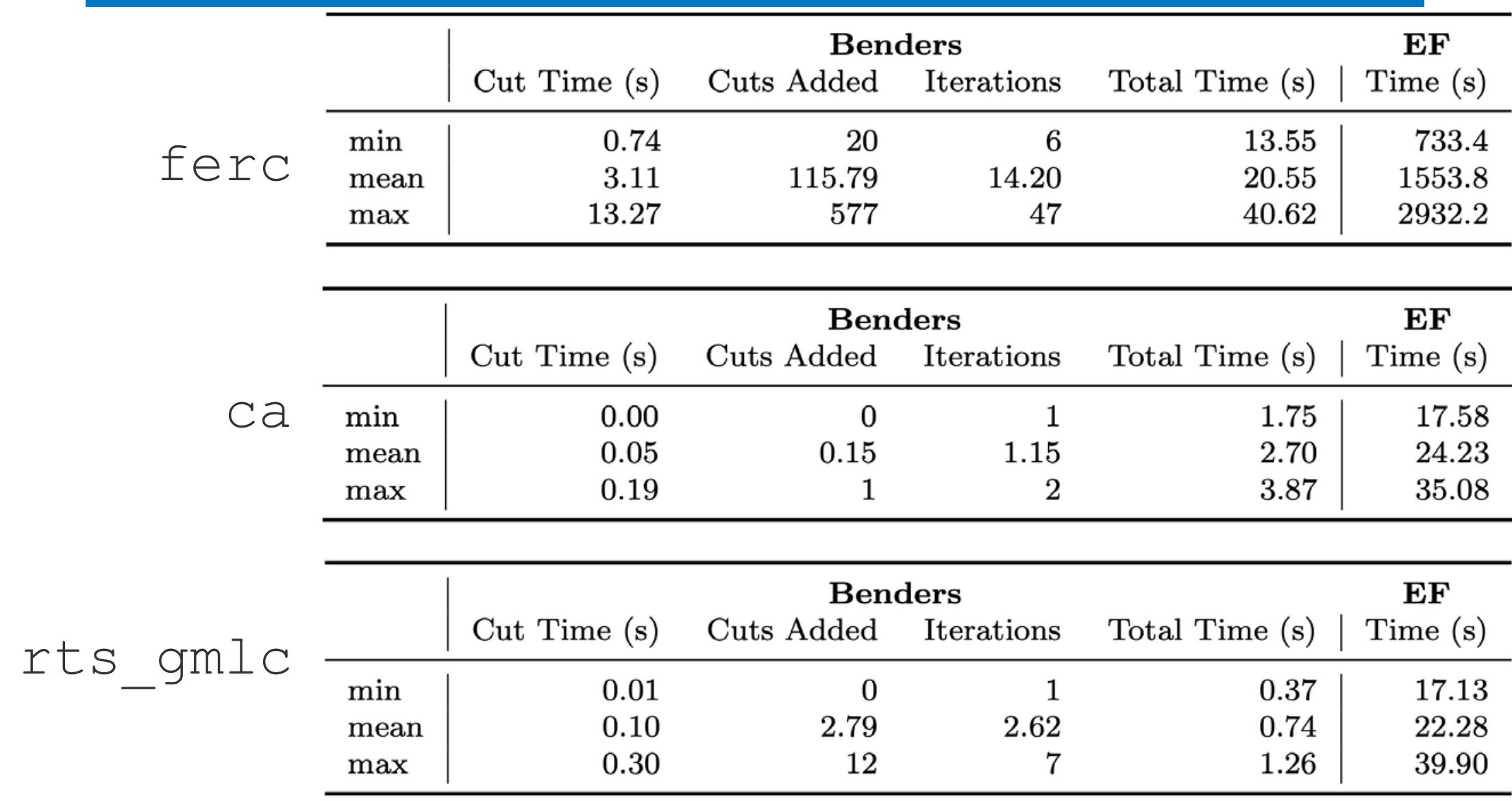




\section{Computational Results: 48-hour Instances}

\begin{tabular}{|c|c|c|c|c|c|c|}
\hline & & & Ben & lers & & $\mathbf{E F}$ \\
\hline & & Cut Time (s) & Cuts Added & Iterations & Total Time (s) & Time (s) \\
\hline & $\min$ & 13.5 & 42 & 5 & 61.1 & * \\
\hline 土erc & mean & 93.4 & 184.6 & 20.2 & 165.0 & * \\
\hline & $\max$ & 480.5 & 1084 & 86 & 658.1 & * \\
\hline & & & Ben & lers & & $\mathbf{E F}$ \\
\hline & & Cut Time (s) & Cuts Added & Iterations & Total Time (s) & Time (s) \\
\hline $\mathrm{ca}$ & $\min$ & 0.00 & 0 & 1 & 5.91 & 317.3 \\
\hline & mean & 1.31 & 0.0 & 1.0 & 11.55 & 537.5 \\
\hline & $\max$ & 4.72 & 0 & 1 & 17.42 & 745.5 \\
\hline & & & Ben & lers & & EF \\
\hline $\mathrm{cm}] \mathrm{c}$ & & Cut Time (s) & Cuts Added & Iterations & Total Time (s) & Time (s) \\
\hline$\perp$ & $\min$ & 0.80 & 0 & 1 & 1.85 & 369.9 \\
\hline & mean & 4.27 & 5.29 & 4.0 & 6.59 & 473.7 \\
\hline & $\max$ & 18.49 & 24 & 16 & 21.34 & 645.5 \\
\hline
\end{tabular}




\section{Computational Results}

\section{LP Size}

- Even though the convex hull formulations used are all representable with a polynomial number of variables and constraints, the associated Primal CHPP can be prohibitively large

- For the 48-hour ferc instances, the EF linear programs have 275 million non-zero constraint matrix elements ( 150 million after presolve)

\section{Benders Decomposition}

- Tight but compact generator formulations (a subject of much research in the past decade) can be useful for approximating convex hulls

- Therefore only few Benders iterations are need in practice 
Analysis of Approximate CHPs

$\overline{ }$ 


\section{ferc energy price differences}

Difference in energy prices in every hour against the EF approach across all 24hour ferc instances.

Whiskers are drawn to cover $99 \%$ of observations.

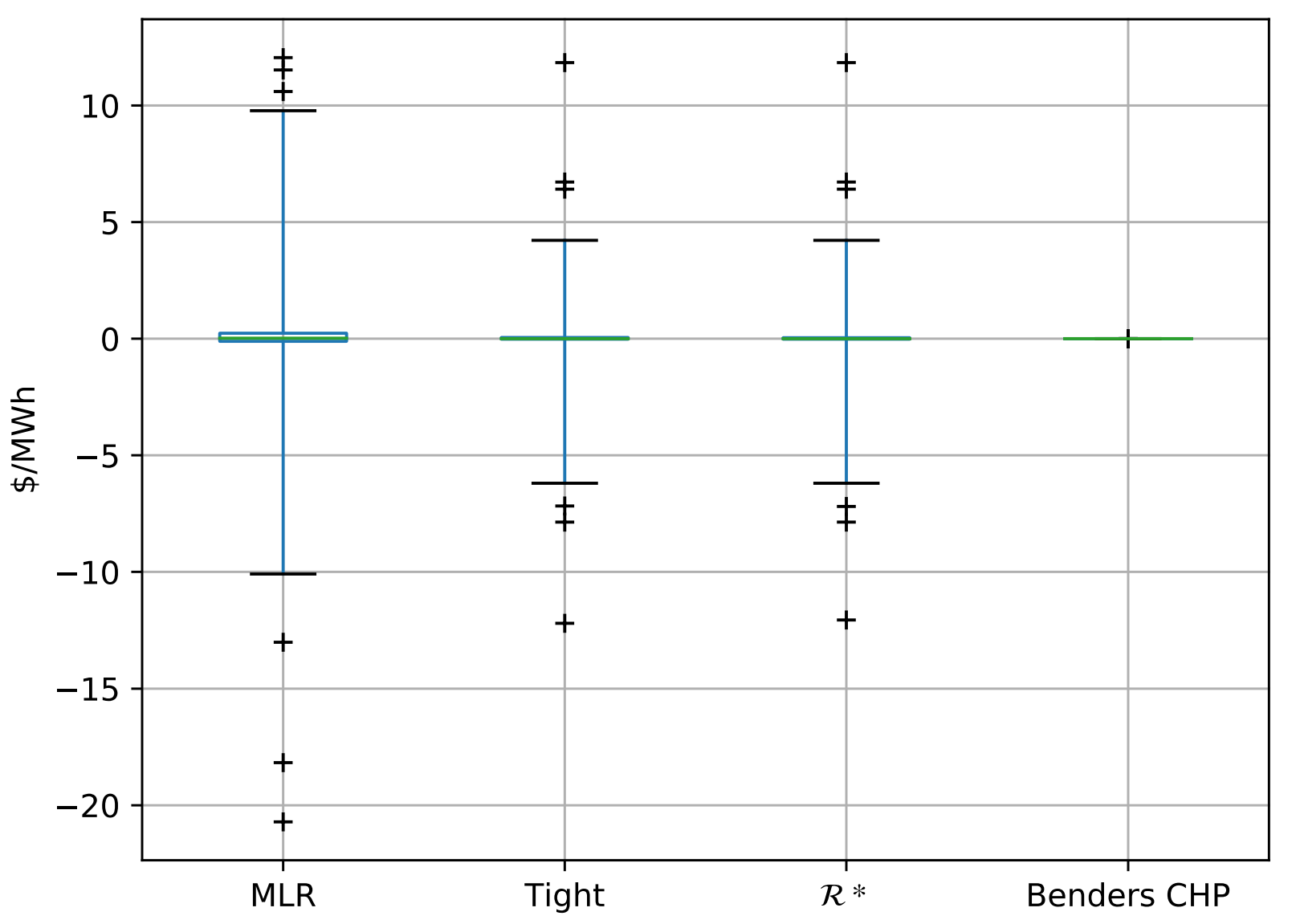




\section{ca energy price}

\section{differences}

Difference in energy prices in every hour against the EF approach across all 24hour ca instances.

Whiskers are drawn to cover $99 \%$ of observations.

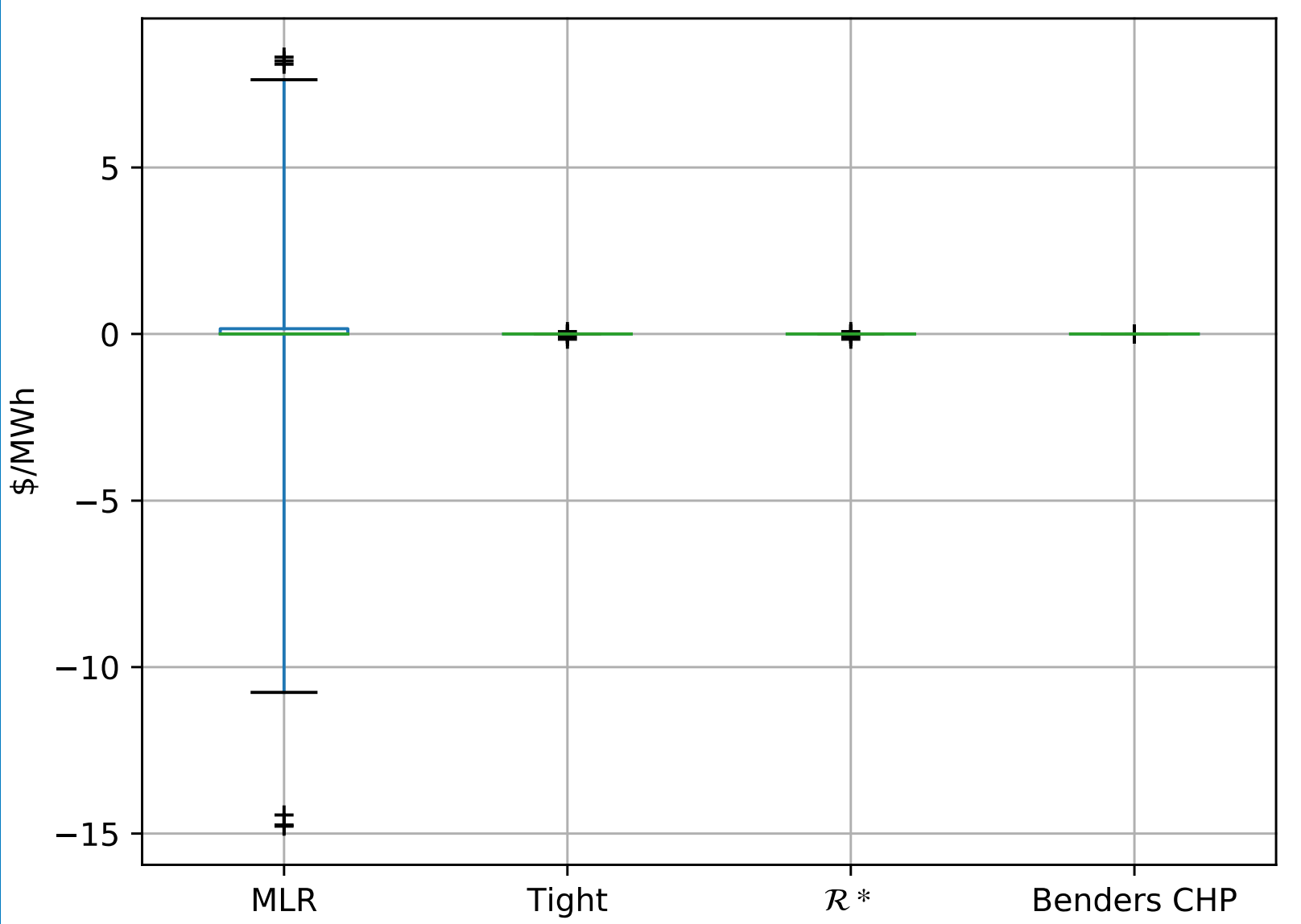


rts_gmlc energy price differences

Difference in energy prices in every hour against the EF approach across all 24hour rts_gmlc instances.

Whiskers are drawn to cover $99 \%$ of observations.

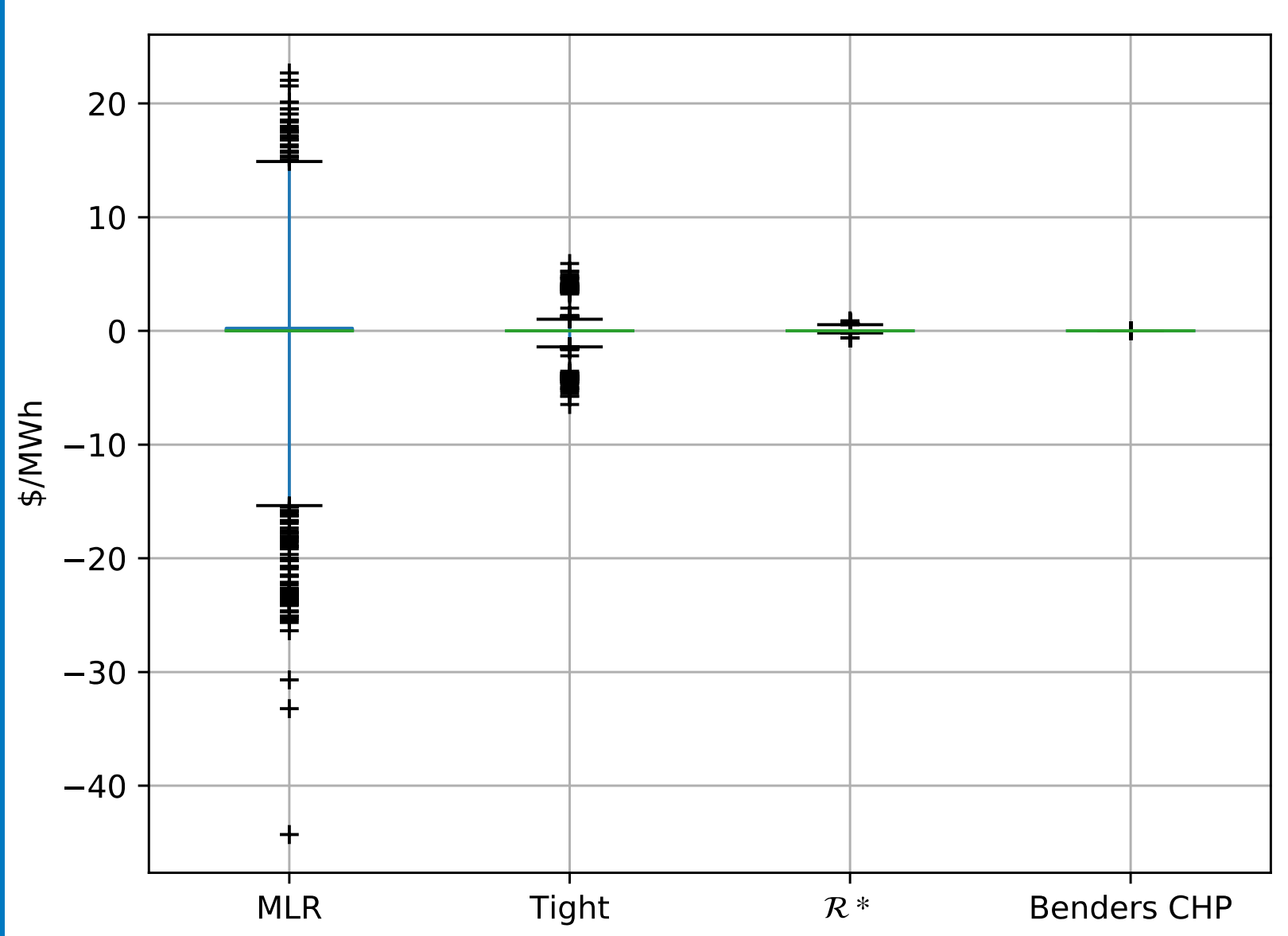


Conclusion 


\section{Conclusion}

- Convex hull prices for energy for large systems can be efficiently computed leveraging the proposed Benders decomposition approach

- "Approximated" convex hull prices can have both small and large errors - the tightness of the approximation is empirically related to the tightness of the generator formulation

- The proposed Benders algorithm can be used as an approximation scheme (terminated after any iteration) if runtime limits are binding

- More work is needed on convex hull representations of generators with complex ancillary service offers and for market participants whose best-known convex hull representation has an exponential number of variables and constraints (e.g., units with maximum daily power, storage). 


\section{Q\&A}

\section{www.nrel.gov}

NREL/PR-2C00-77090

This work was authored in part by the National Renewable Energy Laboratory, operated by Alliance for Sustainable Energy, LLC, for the U.S. Department of Energy (DOE) under Contract No. DE-AC36-08G028308. Funding provided by the U.S. Department of Energy's Office of Electricity Advanced Grid Modeling (AGM) program. The views expressed in the article do not necessarily represent the views of the DOE or the U.S. Government. The U.S. Government retains and the publisher, by accepting the article for publication, acknowledges that the U.S. Government retains a nonexclusive, paid-up, irrevocable, worldwide license to publish or reproduce the published form of this work, or allow others to do so, for U.S. Government purposes. 